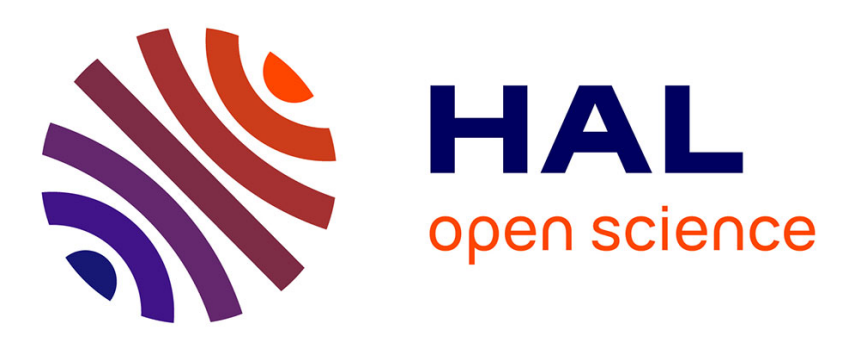

\title{
Simulation du Remplissage des Moules dans les Procédés RTM
}

Juan García, Llanos Gascón, Fernando Sanchez, Francisco Chinesta

\section{To cite this version:}

Juan García, Llanos Gascón, Fernando Sanchez, Francisco Chinesta. Simulation du Remplissage des Moules dans les Procédés RTM. Revue des composites et des matériaux avancés = Journal of Composite and Advanced Materials, 2003, 13 (1), pp.85-98. 10.3166/rcma.13.85-98 . hal-00020759

\section{HAL Id: hal-00020759 \\ https://hal.science/hal-00020759}

Submitted on 17 Feb 2018

HAL is a multi-disciplinary open access archive for the deposit and dissemination of scientific research documents, whether they are published or not. The documents may come from teaching and research institutions in France or abroad, or from public or private research centers.
L'archive ouverte pluridisciplinaire HAL, est destinée au dépôt et à la diffusion de documents scientifiques de niveau recherche, publiés ou non, émanant des établissements d'enseignement et de recherche français ou étrangers, des laboratoires publics ou privés. 


\section{Simulation du Remplissage des Moules dans les Procedes RTM}

\section{Juan A. García*, Llanos Gascón**, Fernando Sánchez*** and Francisco Chinesta*****}

*Universidad Politécnica de Valencia, Dpto. Ing. Mecánica y Materiales, Camino de Vera s/n, E-46022 Valencia (Spain).jugarcia@mcm.upv.es

**Universidad Politécnica de Valencia, Dpto. Matemática Aplicada, Camino de Veras/n, E-46022 Valencia (Spain).llgascon@mat.upv.es

*** Universidad Cardenal Herrera CEU. Escuela Superior de Enseñanzas Técnicas C/ San Bartolomé 55, E-46115 Alfara del Patriarca, Valencia (Spain). sanchezf@uch.ceu.es

**** Laboratoire de Mecanique des Systemes et des Procedes, UMR CNRSENSAM-ESEM, 151 Boulevard de l'Hopital, 75013 Paris (France). francisco.chinesta@paris.ensam.fr

RESUME: Cet article porte sur la discrétisation de l'équation de convection qui gouverne l'évolution de la fonction de présence de la résine dans une simulation à maillage fixe des procédés RTM. Différents limiteurs de flux, décrits dans (García et al., 2003) seront ici analysés. Pour éviter les erreurs numériques introduites par le couplage entre les équations gouvernant la cinématique de l'écoulement et celle qui gouverne l'évolution du domaine occupé par la résine, nous considérons ici un domaine complètement saturé, dans lequel a lieu un écoulement unidirectionnel à débit constant. De cette façon, connaissant le champ de vitesses, la précision de la solution dépendra uniquement de la qualité de la technique de discrétisation utilisée dans l'intégration de l'équation de convection.

MOTS CLES: RTM, Equation de convection, Limiteurs de flux 


\section{Introduction}

García et al., (2003) ont proposé une méthode explicite à maillage fixe pour la simulation des procédés d'injection de résine (RTM). Celle ci découple la résolution des équations du mouvement gouvernant la cinématique de l'écoulement, et la résolution de l'équation de convection gouvernant l'évolution de la fonction de présence de la résine. La cinématique de l'écoulement résulte de la discrétisation de formulation variationnelle faisant intervenir l'équation de Darcy dans le domaine occupé par la résine et l'équation associée à un pseudocomportement (pression nulle) dans la partie du domaine encore vide. Avec cette cinématique calculée au temps $t$, la résolution de l'équation de convection qui gouverne l'évolution de la fonction de présence de la résine nous permet d'actualiser le domaine occupé par la résine au temps $t+\Delta t$. Pour la résolution de cette dernière équation, une technique au second ordre avec des limiteurs de flux à été considérée.

Les limiteurs de flux décrits par Sweby (1984) sont bassés sur des schémas hybrides, qui utilisent des flux au second ordre dans les régions où la solution est suffisamment lisse, pour les réduire au premier ordre à proximité des discontinuités, pour éviter les oscillations numériques caractéristiques des méthodes d'ordre élevé. Ces limiteurs nous permettent de garantir la propriété TVD (Total Variation Diminishing) et donc de résoudre avec précision des problèmes dont la solution peut présenter des discontinuités (Leveque, 1990; Sweby, 1984; Harten, 1983).

Le comportement des différents limiteurs (Minmod, Superbee et Monotonized) a été étudie dans García et al. (2003). Nous avons constaté que dans sa version originale (Sweby, 1984), ils ne peuvent pas être appliqués dans le traitement de certaines situations retrouvées dans la simulation du procédé RTM, concrètement dans le cas d'injection multipoint. Par cette raison, dans García et al. (2003), nous les avons modifiées, et nous avons pu obtenir des résultats excellents. Cependant, jusqu'à présent, nous avons toujours simulé le modèle d'écoulement couplant leur cinématique et l'évolution du domaine occupé par le fluide. Pour pouvoir accéder à une analyse précise des différentes techniques adaptées à la résolution de l'équation de convection, nous considérons dans ce travail un milieu saturé, dans lequel a lieu un écoulement unidirectionnel à débit constant.

\section{Modélisation mécanique}

L'écoulement d'un fluide incompressible dans un milieu poreux peut être décrit à partir de l'équation de conservation de la masse et de la relation entre la vitesse du fluide et le gradient de pression, dont la loi la plus simple fut introduite par Darcy:

- $\quad$ Loi de Darcy 


$$
\underline{v}=-\frac{\underline{\underline{\underline{\underline{K}}}}}{\mu} \nabla p \text { en } \Omega
$$

- Conservation de la masse pour un fluide incompressible

$$
\operatorname{div} \underline{v}=0 \text { en } \Omega
$$

où $\underline{v}$ est le champ des vitesses, $\underline{\underline{K}}$ est le tenseur de perméabilité de la préforme, $\mu$ est la viscosité du fluide et $p$ la pression. Le domaine du moule sera noté par $\Omega, \partial \Omega$ désignant son bord. A chaque instant $t$ nous pouvons écrire

$$
\Omega=\Omega_{f}(t) \cup \Omega_{e}(t)
$$

où $\Omega_{f}(t)$ est la partie du domaine occupée par le fluide (résine) et $\Omega_{e}(t)$ est la partie encore vide, dont l'interface entre les deux, et qui coïncide avec le front de l'écoulement, sera noté par $\Gamma_{f}$. Les conditions aux limites sont données par :

- La composante du gradient de pression normal aux parois du moule est nulle (la vitesse est tangente aux parois).

- La pression ou le débit est imposé dans la buse d'injection.

- La pression est nulle sur le front de l'écoulement.

Pour simuler le procédé d'injection les auteurs ont proposé dans García et al. (2001) une méthode à maillage fixe qui prescrit les équations du mouvement dans la partie du domaine occupé par le fluide et un pseudocomportement (pression nulle) dans la partie vide.

L'actualisation de la partie du moule occupée par la résine est faite par l'intermédiaire de la fonction de présence du fluide $I$, qui est nulle dans la partie vide et qui prend la valeur 1 dans la partie occupée par le fluide:

$$
I(\underline{x}, t)= \begin{cases}1 & \underline{x} \in \Omega_{f}(t) \\ 0 & \underline{x} \notin \Omega_{f}(t)\end{cases}
$$

L'évolution de la fonction $I$ est gouvernée par l'équation de convection

$$
I_{t}+\underline{v} \cdot \nabla I=0
$$

soumise à la condition initiale

$$
I(\underline{x}, t=0)=\left\{\begin{array}{lc}
0 & \underline{x} \in \Omega \\
1 & \underline{x} \in \partial \Omega^{-}
\end{array}\right.
$$

et à la condition de bord sur la buse d'injection

$$
I\left(\underline{x} \in \partial \Omega^{-}, t\right)=1
$$

La formulation variationnelle définie dans le domaine du moule fera donc intervenir la fonction de présence de la résine (García et al., 2003)

$$
\int_{\Omega} f(I) \underline{\underline{K}} \nabla p \cdot \nabla p^{*} d \Omega+\int_{\Omega}(1-f(I)) \alpha(I) p p^{*} d \Omega=0 ; \forall p^{*} \in H_{0}^{1}(\Omega)
$$


où le paramètre $\alpha(I)$ a été introduite afin de garantir l'homogénéité dimensionnelle des deux termes de l'équation [8]; $f(I)$ est une fonction de pondération qui permet d'intégrer le comportement du fluide et le pseudocomportement du vide dans la même formulation variationnelle et $H_{0}^{1}(\Omega)$ est l'espace fonctionnel de Sobolev.

L'algorithme numérique décrit dans García et al. (2001) consiste à résoudre jusqu' au remplissage complet du moule :

1. L'équation [8] à l'aide la méthode des éléments finis, pour obtenir le champ de pressions.

2. L'équation [1] pour obtenir le champ de vitesses.

3. L'équation [5] pour actualiser le domaine occupé par le fluide.

Une des difficultés majeures dans la simulation du procédé RTM est liée précisément à l'actualisation de la fonction de présence de la résine $I$ à cause du caractère hyperbolique de l'équation qui gouverne leur évolution ([5]).

Un grand nombre de stratégies numériques adaptées à ce caractère ont été proposées ces dernières années. La plus part d'entre elles sont caractérisées par la propriété TVD qui permet de garantir l'absence d'oscillations numériques même en présence de discontinuités de la solution. García et al. 2003 ont proposé l'utilisation d'une méthode au second ordre avec limiteurs de flux qui opère sur un maillage composé de triangles. La discrétisation de l'équation [5] conduit à :

$$
I_{i}^{n+1}=I_{i}^{n}-\frac{\Delta t}{A(i)} \sum_{j=1}^{3} l_{i j} \hat{f}_{i j} \cdot \underline{n}_{i j}
$$

où $A(i)$ est l'aire de l'élément $i, j$ représente un triangle voisin, $\underline{n}_{i j}$ le vecteur unitaire normal à l'arête commune entre les triangles $i$ et $j$ (dirigé vers $j$ ) et $l_{i j}$ est la longueur de cette arrête. La définition des schémas du premier et second ordre sont obtenus en remplaçant $\hat{f}_{i j} \cdot \underline{n}_{i j}$ dans l'équation [9] par : (García et al., 2003)

$$
\begin{aligned}
& \hat{f}_{i j}^{U P} \cdot \underline{n}_{i j}=\frac{1}{2}\left\{\left(\underline{f}_{i}+\underline{f}_{j}\right) \underline{n}_{i j}-\operatorname{sign}\left(\cos \left(\theta_{i j}\right)\right)\left(\underline{f}_{j}-\underline{f}_{i}\right) \underline{n}_{i j}\right\} \\
& \underline{\hat{f}}_{i j}^{S W} \cdot \underline{n}_{i j}=\underline{\hat{f}}_{i j}^{U P} \cdot \underline{n}_{i j}+\frac{1}{2} \chi\left(r_{i j}\right)\left(\operatorname{sign}\left(\cos \left(\theta_{i j}\right)\right)-\frac{\Delta t}{d_{i j}} \underline{v}_{i j} \cdot \underline{n}_{i j}\right)\left(\underline{f}_{i}+\underline{f}_{j}\right) \underline{n}_{i j}
\end{aligned}
$$

où $f=I \underline{v}, \theta_{\mathrm{ij}}$ est l'angle entre $\underline{\mathrm{n}}_{\mathrm{ij}}$ et le vecteur vitesse $\underline{v}_{\mathrm{j}}$, et $d_{i j}$ représente la distance entre les barycentres des triangles $i$ et $j$. L'exposante $U P$ est associé au schéma de premier ordre décentré en amont (upwind) tandis que l'exposant $S W$ est employé pour noter les schémas du second ordre. En ce qui concerne $r_{i j}$, nous proposons de définir leur valeur en comparant les valeurs des solutions numériques entre deux éléments voisins (du point de vue de la direction de l'écoulement), avec $\chi(r)$ le limiteur de flux, dont la valeur dépend de la régularité de la solution. Le cas $\chi(r)=1$ conduit à l'extension du schéma de Lax-Wendroff sur des maillages d'éléments 
finis, et le cas $\chi(r)=0$ représente un décentrage en amont. Dans ce qui suit, nous nous intéresserons aux limiteurs de flux suivants :

$$
\begin{array}{ll}
\chi_{M M}(r)=\max \{0, \min \{r, 1\}\} & \text { Minmod } \\
\chi_{S B}(r)=\max \{0, \min \{2 r, 1\}, \min \{r, 2\}\} & \text { Superbee } \\
\chi_{M C}(r)=\max \{0, \min \{2 r, 2,(1+r) / 2\}\} & \text { Monotonized Centered }
\end{array}
$$

Ces limiteurs ont pour mission d'annuler le terme de flux à l'origine de la précision de second ordre à proximité des discontinuités, préservant la propriété TVD, et donc l'absence des oscillations numériques caractéristiques des schémas du second ordre. Ces différents limiteurs seront comparés dans les exemples numériques qui suivent.

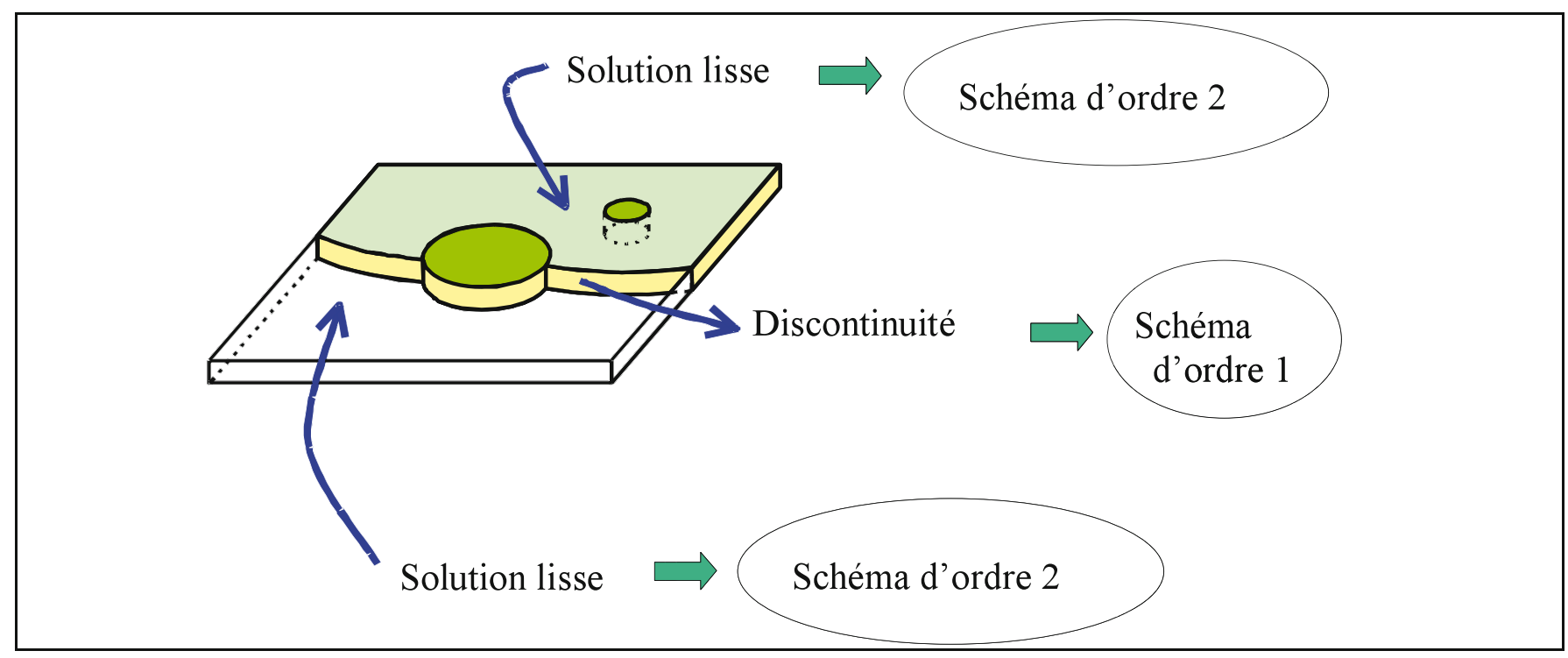

Figure 1. Zones d'application des schémas du premier et second ordre

\section{Exemples numériques}

Nous considérons un milieu poreux saturé où un écoulement unidirectionnel à débit constant a lieu. Dans cette situation très simplifiée, nous pouvons calculer la solution exacte concernant la position d'un hypothétique front transporté par l'écoulement, et donc évaluer la précision des différentes techniques numériques. Le moule est représenté dans la figure 2 , où toutes les dimensions sont en $\mathrm{mm}$. Une résine à viscosité de $0.1 \mathrm{~Pa}$.s est injectée avec une vitesse $v_{i}$ de $10^{-1} \mathrm{~m} / \mathrm{s}$. La préforme est assumée isotrope, de perméabilité $10^{-8} \mathrm{~m}^{2}$. Ainsi la pression exacte peut être évaluée à partir de l'équation de Darcy :

$$
p=v_{i} \frac{\mu}{K}\left(L_{m}-x\right)=10^{6}(1-x)
$$

où $L_{m}$ est la longueur du moule et $x$ représente la position par rapport au bord d'entrée du fluide. 


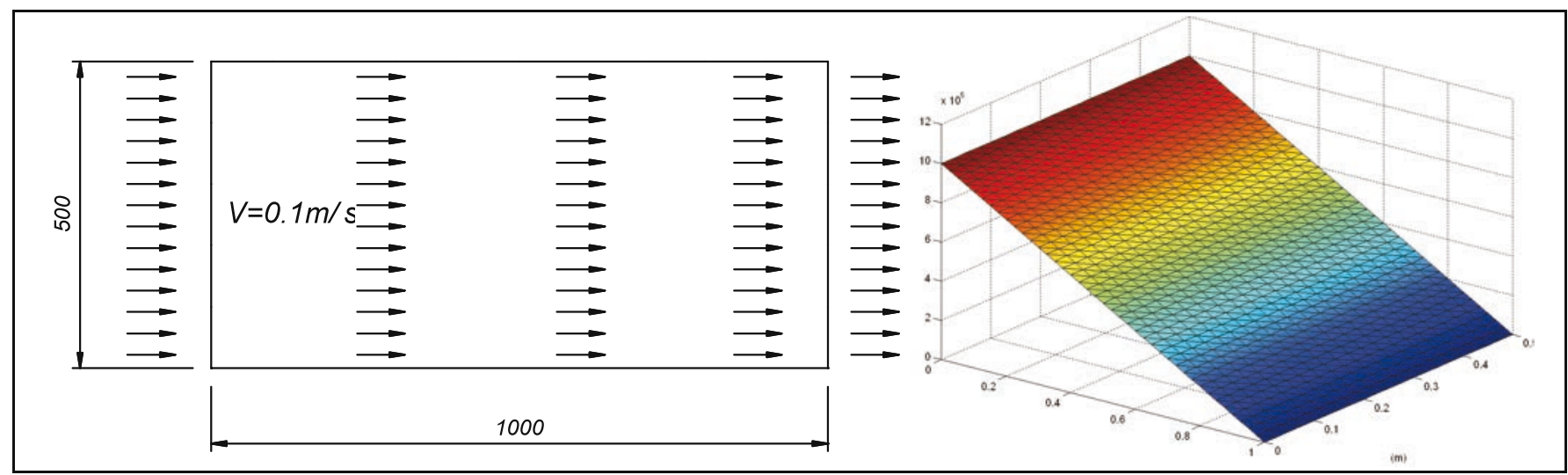

Figure 2. Ecoulement unidirectionnel

Les figures 3, 4, 5 et 6 montrent pour différentes tailles de maillage, la comparaison entre les différents schémas numériques (premier ordre, Lax-Wendroff, minmod, superbee et monotonized) appliqués à l'actualisation de la fonction de présence de la résine à $t=3.6 \mathrm{~s}$. Les maillages considérés comportent 1600 éléments (figure 3), 400 éléments (figure 4), 240 éléments (figure 5) et 100 éléments (figure $6)$.

Les solutions exactes et numériques concernant cette fonction sont également représentées dans tous les éléments du maillage (pour le maillage le plus fin), dans les figures 7, 8 et 9. Comme on peut l'apprécier, l'utilisation du schéma de LaxWendroff ou du schéma du premier ordre produisent les pires solutions. Dans le premier cas, à cause des oscillations numériques, et dans le deuxième à cause de la surdiffusion caractéristique des schémas décentrés du premier ordre. Les meilleurs résultats ont été obtenus avec les limiteurs monotonized et superbee, où la discontinuité a été bien prise en compte indépendamment de la taille du maillage.

\section{Discussion}

Dans les figures 10 et 11 nous avons représenté la différence entre les volumes de fluide exacte et numérique obtenue avec les différentes techniques de discrétisation, pour deux différentes maillages. L'erreur dans chaque élément a été définie de la manière suivante

$$
e_{i}=I_{i}(\text { calculated })-I_{i}(\text { exact })
$$

Comme l'on peut constater, l'erreur est minimum quand les limiteurs de flux superbee ou monotonized sont utilisés. L'analyse de convergence est faite en considérant la norme $\mathrm{L}^{2}$. Les résultats montrés dans la figure 12 nous permettent d'affirmer que la vitesse de convergence est similaire pour toutes les techniques. 


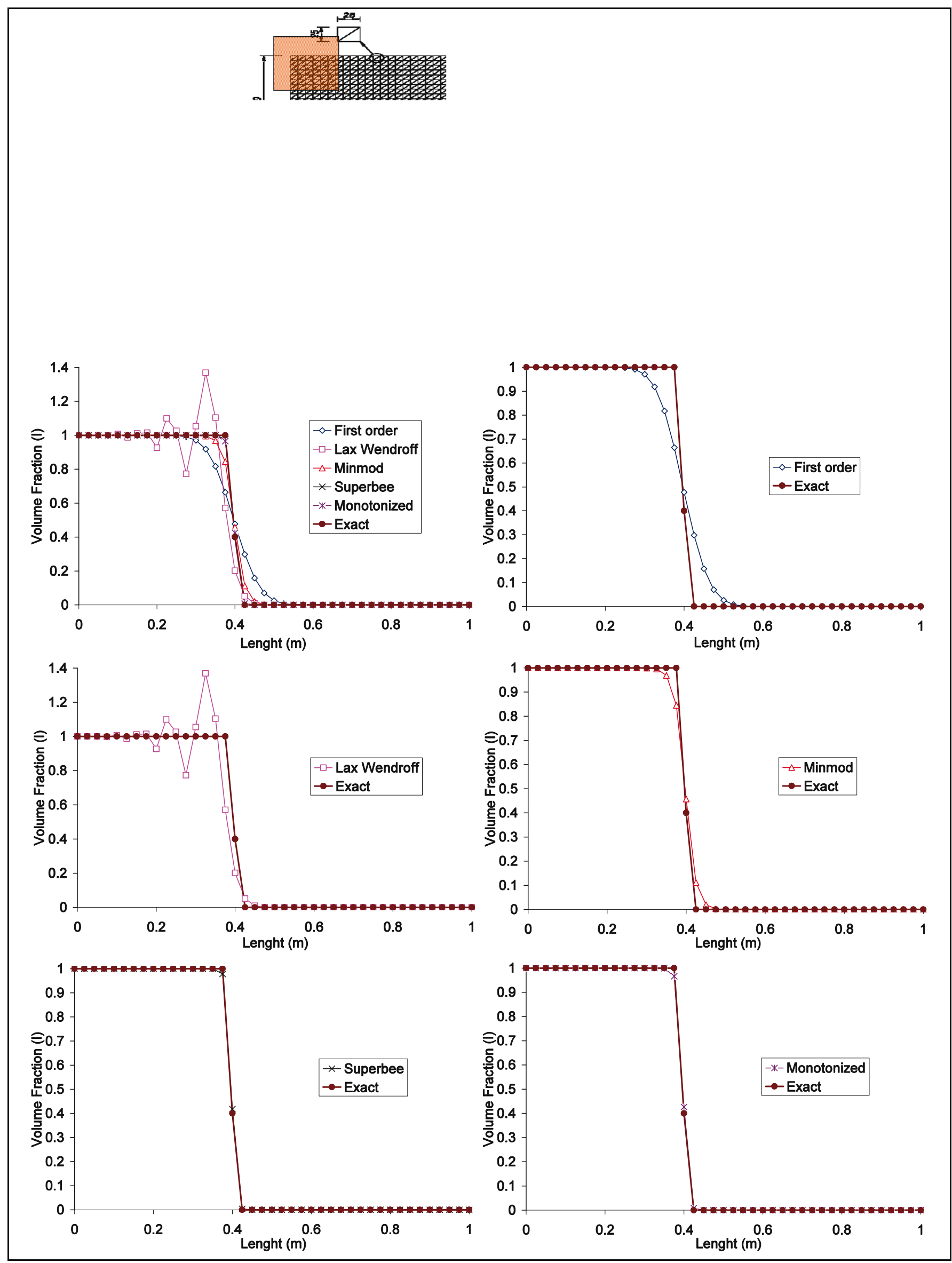

Figure 3. Comparaison des différents schémas pour un maillage de 1600 éléments 


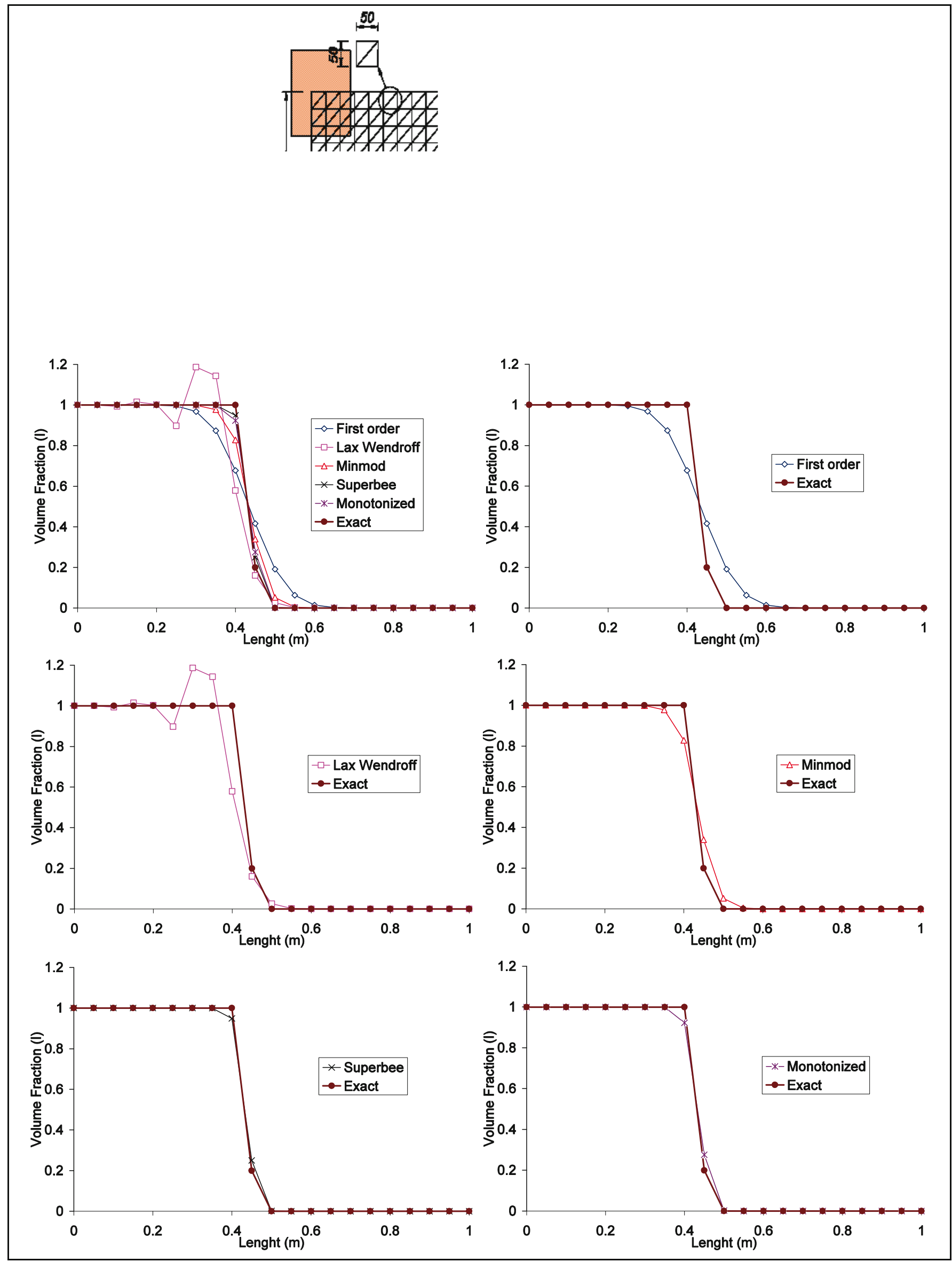

Figure 4. Comparaison des différents schémas pour un maillage de 400 éléments 

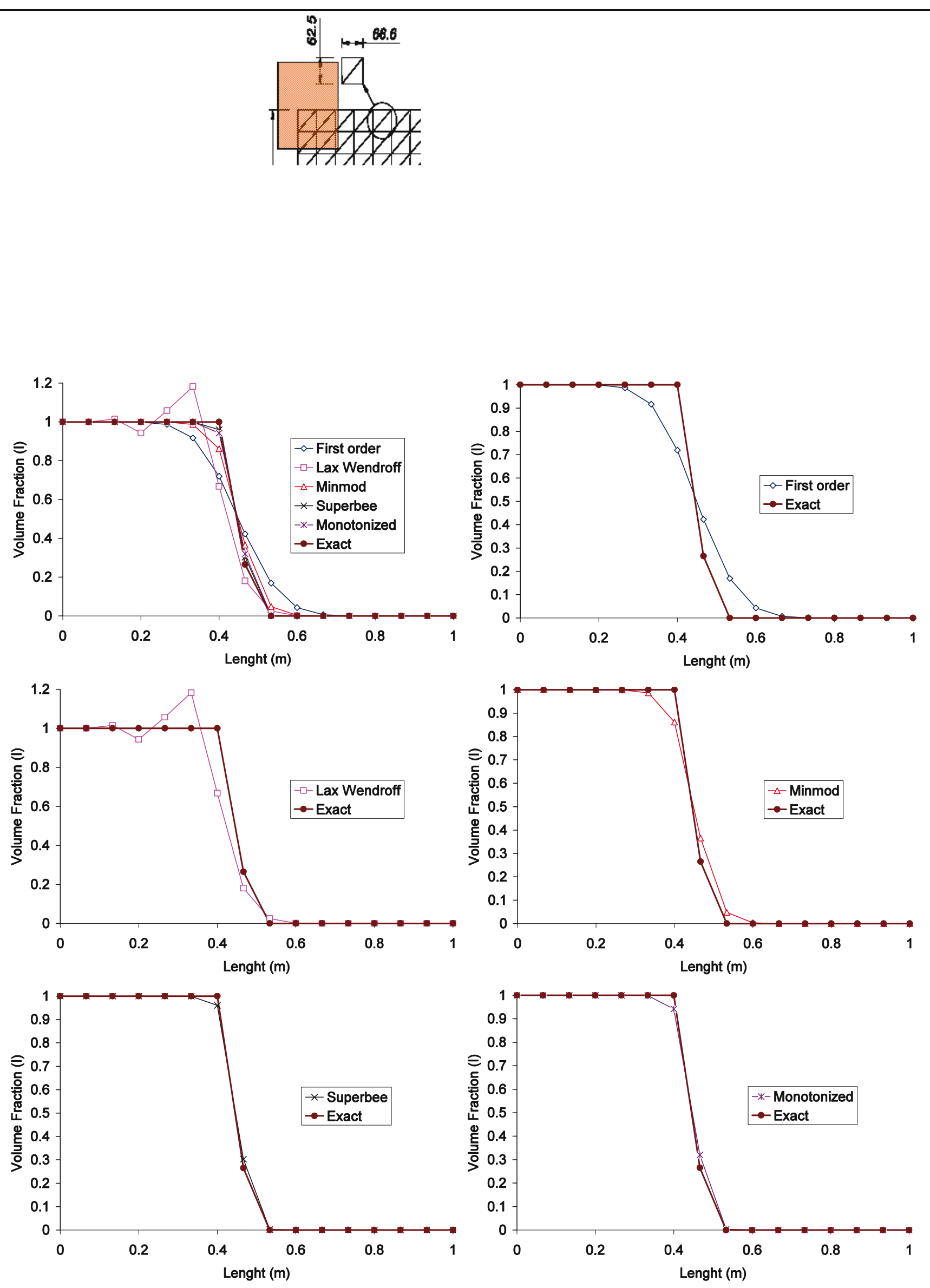

Figure 5. Comparaison des différents schémas pour un maillage de 240 éléments 

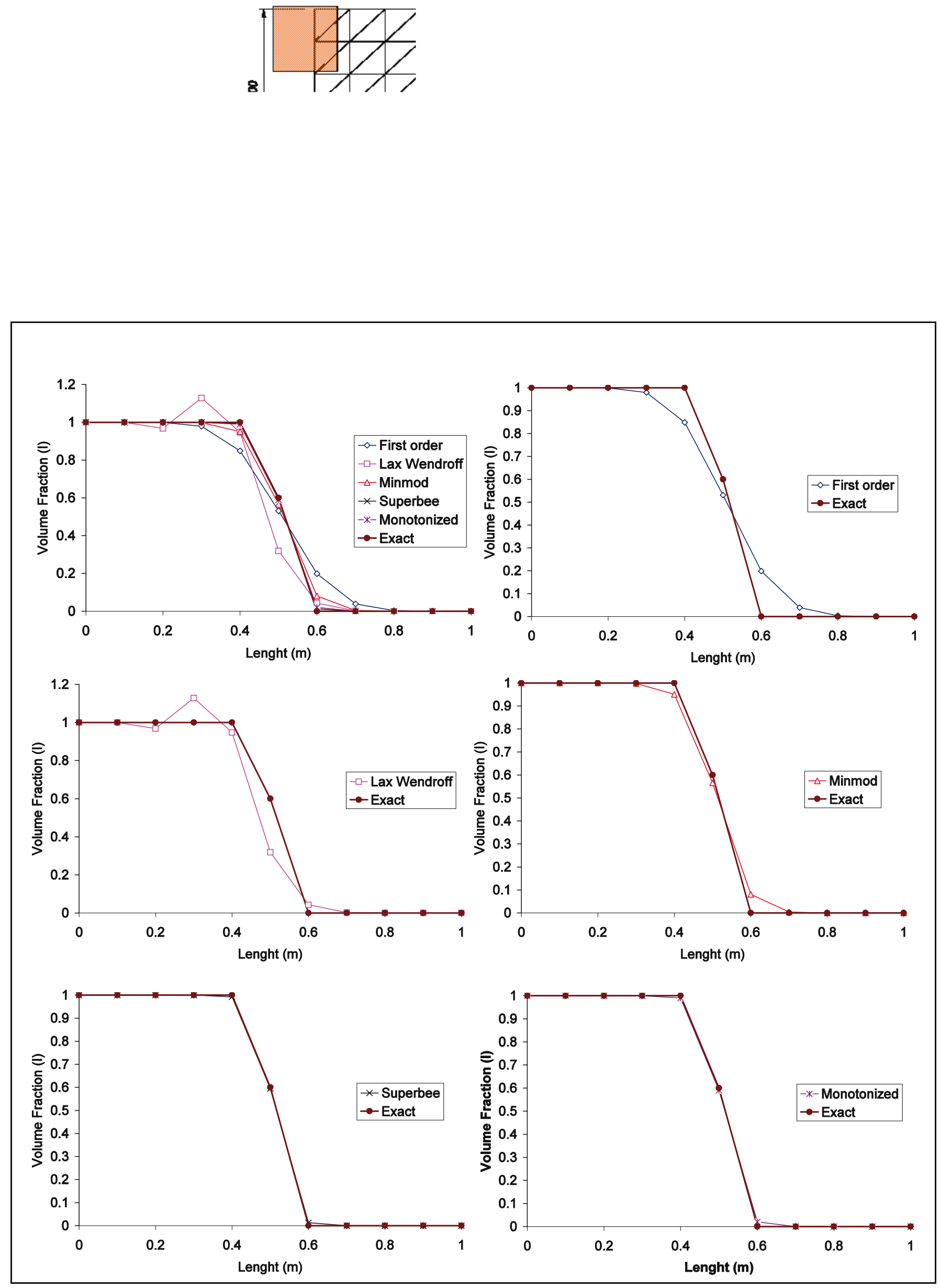

Figure 6. Comparaison des différents schémas pour un maillage de 100 éléments 


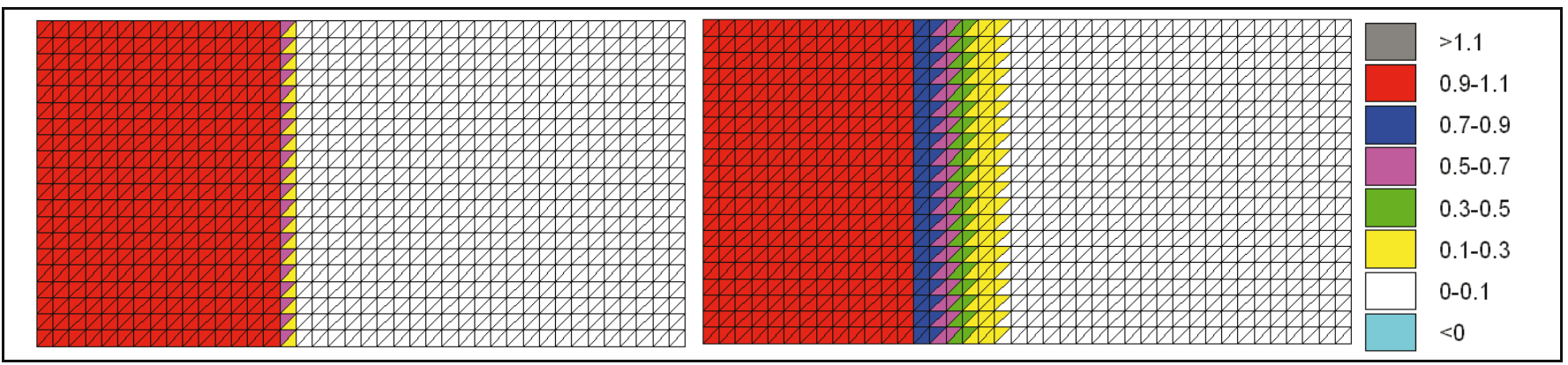

Figure 7. Solution exacte (à gauche) et numérique du premier ordre (à droite) pour $3.6 \mathrm{~s}$.

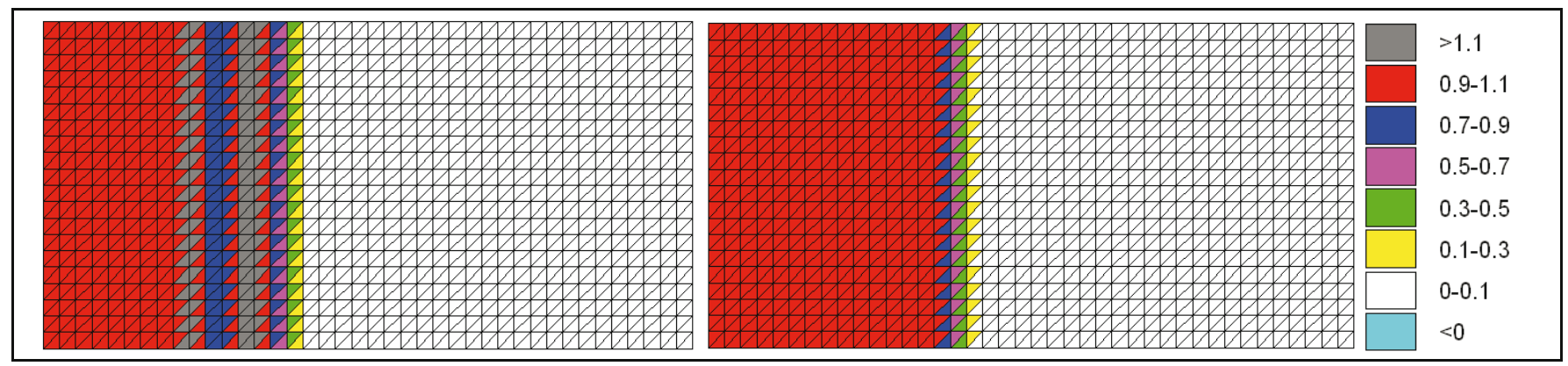

Figure 8. Solution Lax-Wendroff (à gauche) et avec un limiteur minmod (à droite) pour $3.6 \mathrm{~s}$.

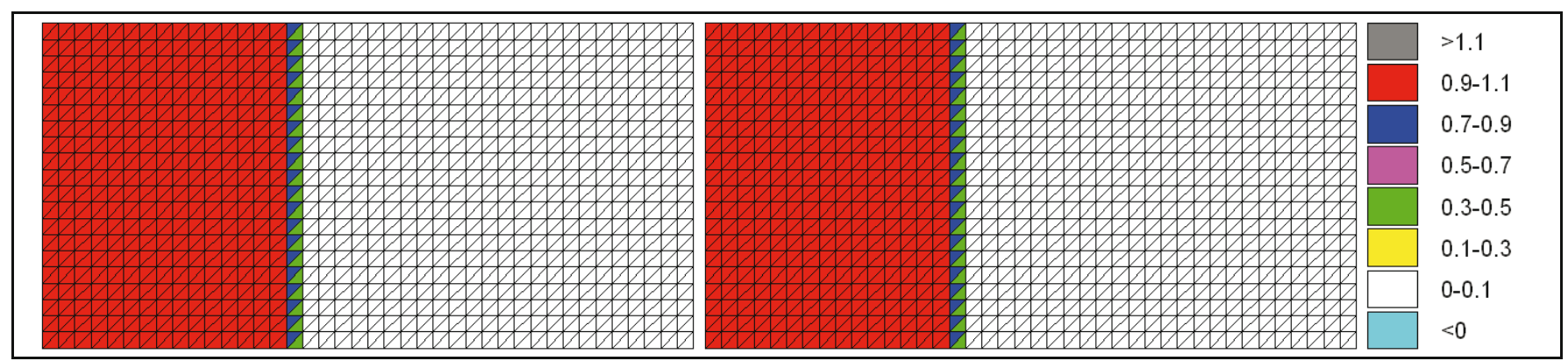

Figure 9. Solution avec le limiteur superbee (à gauche) et avec le limiteur monitonized (à droite) pour $3.6 \mathrm{~s}$. 


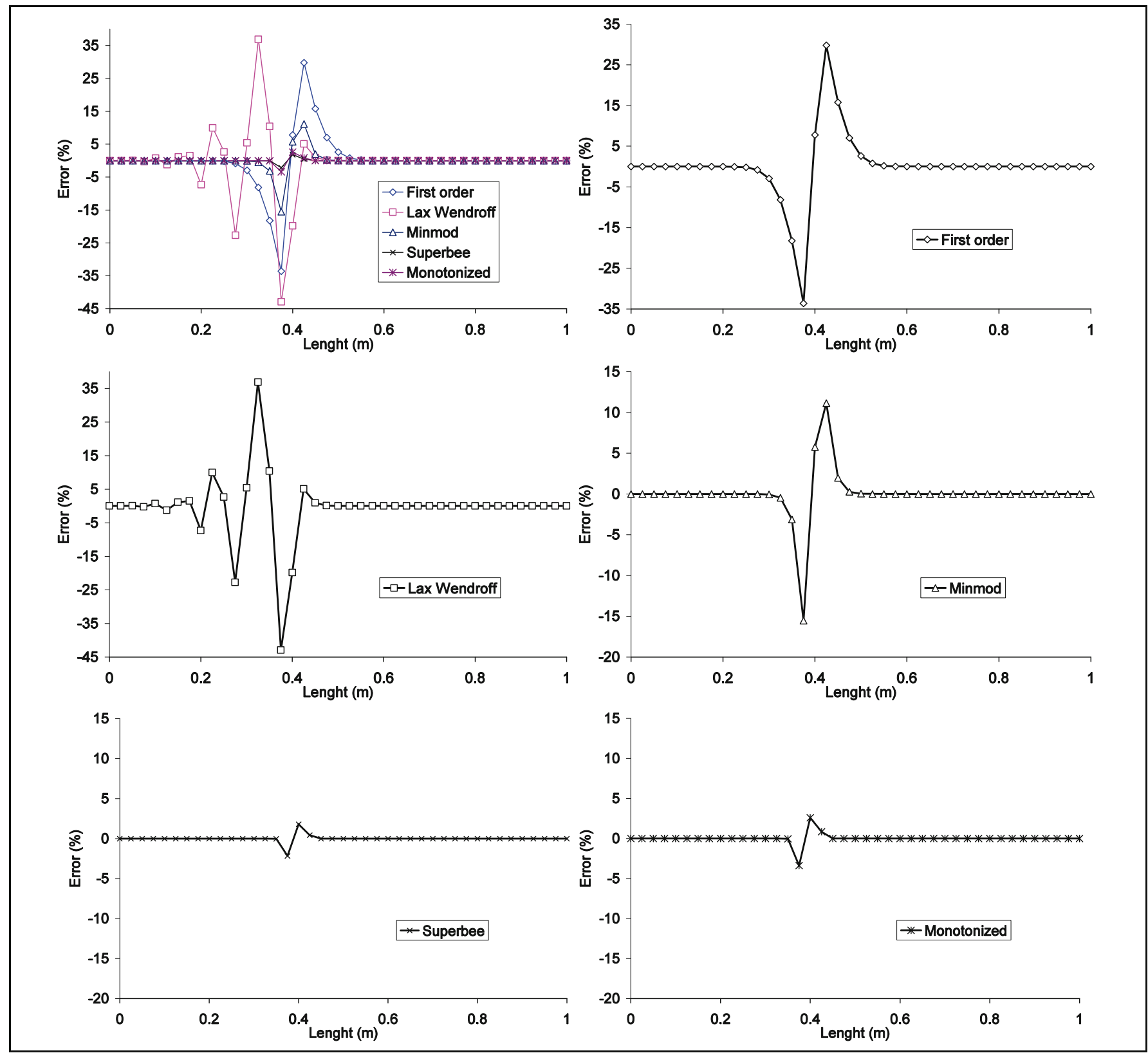

Figure 10. Erreur pour $t=3.6$ s. (1600 éléments) 


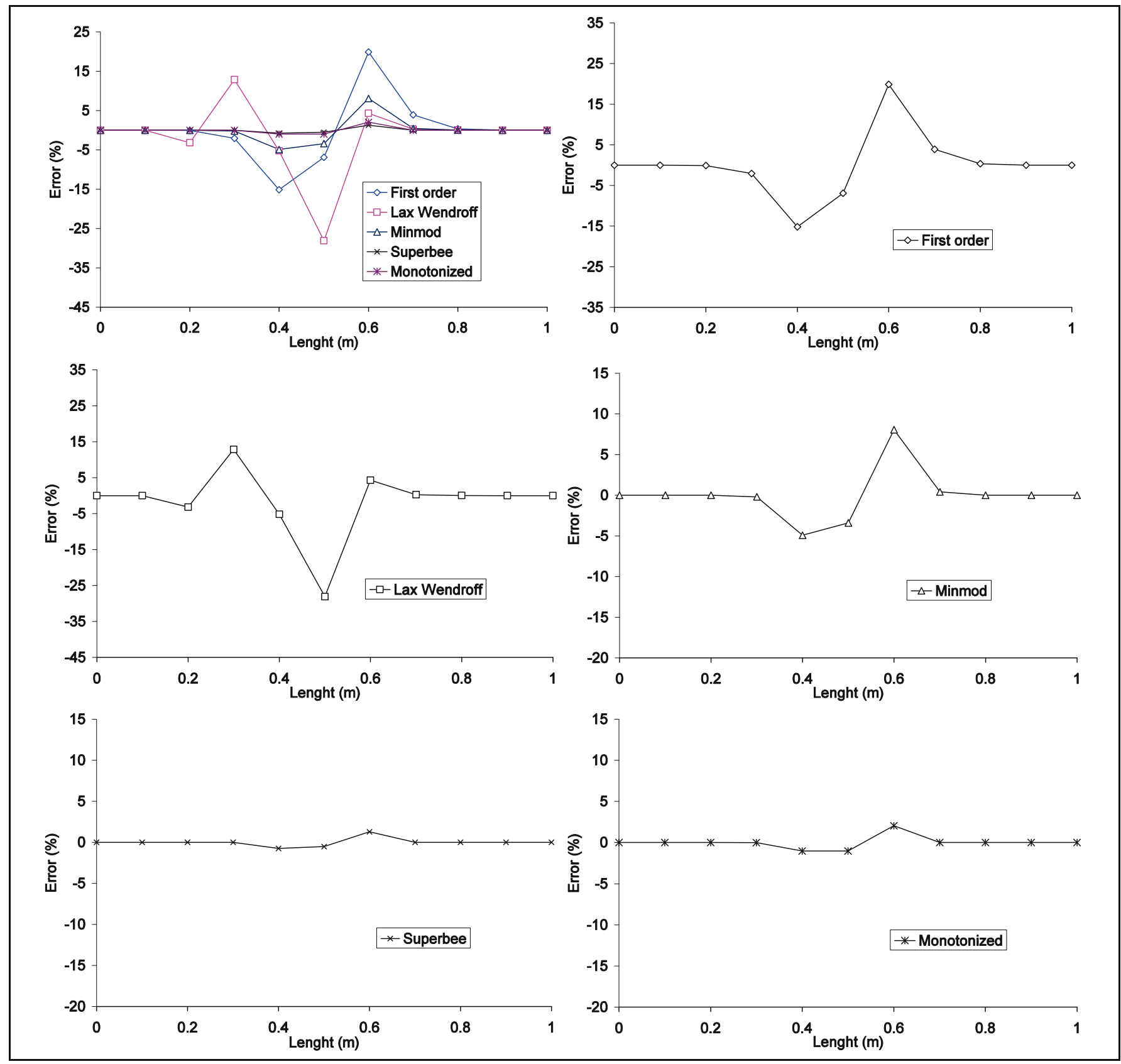

Figure 11. Erreur pour $t=3.6 \mathrm{~s}$. (100 éléments)

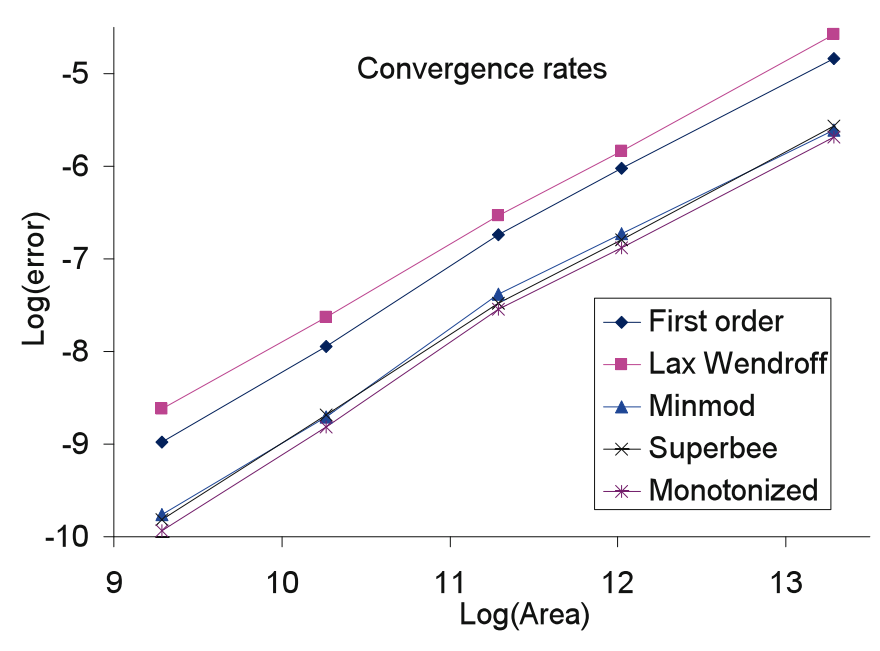

Figure 12. Analyse de convergence 


\section{Conclusions}

Dans cet article nous avons comparé le comportement de différents limiteurs de flux (minmod, superbee et monotorized) utilisés dans l'actualisation de la fonction de présence du fluide dans la simulation du procédé RTM. De cette analyse nous avons conclu que les meilleurs résultats sont obtenus en utilisant le limiteur de flux superbee. Ces résultats sont en cohérence avec les résultats déjà obtenus par le modèle couplé (Garcia et al., 2003).

\section{Remerciements}

Ces recherches ont été finances dans le cadre des aides du Ministerio de Ciencia y Tecnología (MCYT) et du Fondo Europeo de Desarrollo Regional (FEDER), (projet DPI2001-2792).

\section{Références}

García J.A., Gascón Ll., Chinesta F., "A Fixed Mesh Numerical Method for Modelling the Flow in Liquid Composites Moulding Processes Using a Volume of Fluid Technique", Compt Method Appl M. 192 (7-8) (2003) 877-893.

García J.A., Gascón Ll., Chinesta F. "A Second Order TVD Scheme for the Calculation of the Volume-Fraction Evolution in Porous Media Flow Simulation". International Conference of Material Forming (ESFORM), vol. 1, pp. 23-26, (2001).

Harten A., "High Resolution Schemes for Hyperbolic Conservation Laws", J. Comput. Physic. 49 (1983) 357-393.

Leveque R., "Numerical Methods for Conservation Laws" (Birkhäuser, Basel/Boston/Berlin, 1990).

Sweby P.K., "High Resolution Schemes Using Flux Limiters For Hyperbolic Conservation Laws", SIAM J. Numer. Anal. (1984) 995-1011. 